\title{
28. On Orientable Manifolds of Dimension Three
}

\author{
By Hiroshi Yamasuge and Yoshihiro Saito \\ (Comm. by K. KunUGI, M.J.A., March 12, 1960)
}

Let $M$ be a closed orientable differentiable manifold of dimension 3 and $f$ be a function on $M \times I$ where $I=[-1,1]$. Let $x_{i}(i=1,2,3)$ be a local coordinate system of $M$ and $t$ be the parameter varying on $I$. We write $f_{t}$ instead of $f$ when we consider that $f$ is a function on $M$ for fixed $t$. A point at which every first derivative of $f_{t}$ with respect to $x_{i}$ vanishes is called stational point and it is called ordinary stational point or super stational point according as: $\operatorname{det}\left(\frac{\partial^{2} f}{\partial x_{i} \partial x_{j}}\right) \neq 0$ or $\operatorname{det}\left(\frac{\partial^{2} f}{\partial x_{i} \partial x_{j}}\right)=0$.

If the origin $x_{i}=0(i=1,2,3)$ is an ordinary stational point of $f_{0}$, in a neighborhood of this point $f_{t}$ becomes

where $|t|$ is small and $\operatorname{det}\left(a_{i j}(0)\right) \neq 0$.

$$
f_{t}=a(t)+\Sigma a_{i j}(t) x_{i} x_{j}
$$

And if $x_{i}=0$ is a super stational point of $f_{0}$, by a suitable coordinate system $f_{t}$ is represented as

$$
f_{t}=c+c_{0} t+\sqrt{-c_{1} t} x_{1}^{2}+c_{2} x_{2}^{2}+c_{3} x_{3}^{2}+\frac{1}{3} x_{1}^{3}
$$

where $x_{2}=o(\sqrt{|t|})$ and $x_{3}=o(\sqrt{|t|})$. Here we can assume that all $c_{\nu}(\nu=0$, $1,2,3)$ are not 0 . Hence for a small $|t|$ we have two stational points $(0,0,0)$ and $\left(-2 \sqrt{-c_{1} t}, 0,0\right)$ of $f_{t}$. At the point $(0,0,0)$ or $\left(-2 \sqrt{-c_{1} t}, 0\right.$, $0) f_{t}$ is represented as $c+c_{0} t+\sqrt{-c_{1} t} x_{1}^{2}+c_{2} x_{2}^{2}+c_{3} x_{3}^{2}$ or $c+c_{0} t-\sqrt{-c_{1} t}\left(x_{1}\right.$ $\left.+2 \sqrt{-c_{1} t}\right)^{2}+c_{2} x_{2}^{2}+c_{3} x_{3}^{2}$ where all $c_{\nu}(\nu=0,1,2,3)$ are not zero. We call a stational point to be type $(\mu)$ if the non-degenerate diagonal quadratic form in the Taylor's expansion of $f_{t}$ at this point has $\mu$ negative terms.

Suppose the above origin is type $(\mu)$ then $\left(-2 \sqrt{-c_{1} t}, 0,0\right)$ is type $(\mu+1)$ and we call the super stational point $(0,0,0)$ of $f_{0}$ to be type $(\mu, \mu+1)$ or $(\mu+1, \mu)$ according as $c_{1}<0$ or $c_{1}>0$. We see easily that values of $t$ on the locus of stational points take the minimums or the maximums at points of type $(\mu, \mu+1)$ or $(\mu+1, \mu)$.

Let $D$ and $D^{\prime}$ be two solid spheres with $n$ holes as Fig. 1 and $\sigma$ a homeomorphism of $\partial D$ to $\partial D^{\prime}$ and $D_{\sigma}^{\smile} D^{\prime}$ the manifold defined by identifying $\partial D$ and $\partial D^{\prime}$ by $\sigma$.

Now we consider the necessary and sufficient condition so that $D_{\sigma}^{\smile} D^{\prime}$ is diffeomorphic with $D_{\tau}^{\smile} D^{\prime}$. Clearly we can construct a function $g$ on $D_{\sigma}^{\smile} D^{\prime}$ satisfying the following conditions.

a) $g<0$ in $D-\partial D, g=0$ on $\partial D$ and $g>0$ in $D^{\prime}-\partial D^{\prime}$. 


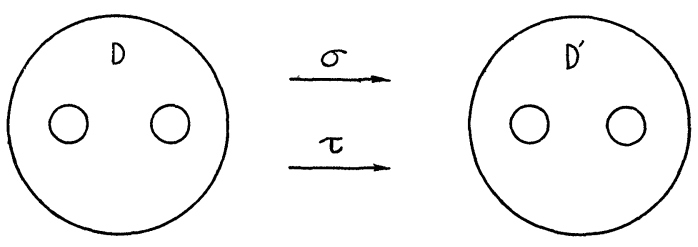

Fig. 1

b) In $D g$ has one stational point of type (0) and $n$ stational points of type (1) and in $D^{\prime} g$ has $n$ stational points of type (2) and one stational point of type (3). Similarly we construct a function $h$ on $D_{\tau}^{\smile} D^{\prime}$ satisfying the above conditions. Put $M=D_{\sigma}^{\smile} D^{\prime}$ and $N=D_{\tau}^{\smile} D^{\prime}$ and let $u$ be a diffeomorphism of $M$ on $N$. From now on we write $M \simeq N$ when $M$ is diffeomorphic with $N$.

Now we consider the function $f_{t}(p)=\frac{1-t}{2} g(p)+\frac{1+t}{2} h(u p)$, for $p \in M$. Then from the above we have

Lemma 1. In the locus of stational points of $f_{t}$ the number of the points of type $(1,2)$ is equal to the number of the points of type $(2,1)$.

If $x_{i}=0 \quad(i=1,2,3)$ is a super stational point of $f_{0}$ and $|t|$ is sufficiently small we can reform $f_{t}$ a little in a neighborhood $U$ containing $(0,0,0)$ and $\left(-2 \sqrt{-c_{1} t}, 0,0\right)$ so that $f_{t}$ has no stational point in $U$. And conversely if $f_{t}$ is regular in $U$ we can reform $f_{t}$ a little in $U$ so that $f_{t}$ has two stational points as mentioned above. From these by reforming $f$ along suitable pathes we can change the locus of stational points so that we have

Lemma 2. For the function $f$ there exists a function $\bar{f}$ satisfying the following properties:

a) For every positive number $\varepsilon$ we can take a sufficiently small positive number $\delta$ so that $\left|\bar{f}_{-1+s}-f_{-1+s}\right|<\varepsilon$ and $\left|\bar{f}_{1-s}-f_{1-s}\right|<\varepsilon$ for $0 \leqq s \leqq \delta$.

b) The t-coordinates of all stational points of type $(1,2)$ or $(2,1)$ are -1 or 1 and the values of $\bar{f}$ at these points are always 0 .

Introduce in $M$ a Riemannian metric

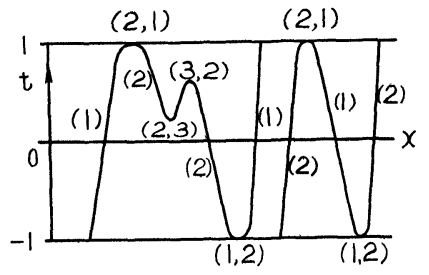

Fig. 2 and consider stream lines which are normal on every equi-potential surface of $\bar{f}_{t}$. If necessary by reforming $\bar{f}_{t}$ in tubular neighborhoods of stream lines which flow out from or flow into stational points of type (1) or (2) and in neighborhoods of stational points of type (0) or (3), we get

Lemma 3. We can make $\bar{f}$ in Lemma 2 have the property $c$ ) 
besides $a$ ) and $b)$ :

c) The values of $\bar{f}$ at stational points of type $(0),(1),(0,1)$ or $(1,0)$ are always negative and the values of $\bar{f}$ at stational points of type (2), (3), $(2,3)$ or $(3,2)$ are always positive.

Let $m$ be the number of stational points of type $(1,2)$ and $G_{k}$ $(k=1, \cdots, m)$ small solid cylinders in $D$. Since by $\sigma \Sigma \partial G_{k} \partial D$ is identified to $\sigma\left(\Sigma \partial G_{k} \partial D\right) \subset \partial D^{\prime}$ we cut out $\Sigma G_{k}$ from $D$ and bring it to $\partial D^{\prime}$, write it $\Sigma \sigma G_{k}$, and paste $\Sigma \partial G_{k} \partial D$ on $\sigma\left(\Sigma \partial G_{k \frown} \partial D\right)$ by $\sigma$.

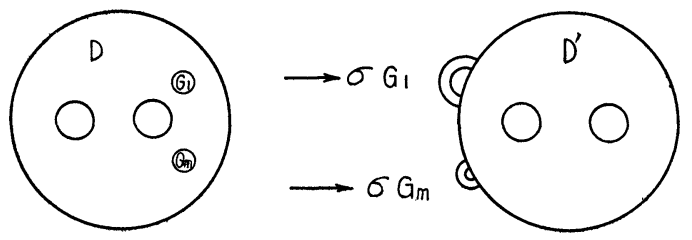

Fig. 3

Denote by $\bar{\sigma}$ the identifying map of $\partial\left(D-\Sigma G_{k}\right)$ to $\partial\left(D^{\prime} \smile \Sigma \sigma G_{k}\right)$ obtained from $\sigma$ by the above operation.

Putting $M_{\delta}=\left\{p \mid \bar{f}_{\delta}(p) \leqq 0\right\}$ and $M_{\delta}^{\prime}=\left\{p \mid \bar{f}_{\delta}(p) \geqq 0\right\}$, we have $M_{\delta} \simeq D$ $-\Sigma G_{k}, M_{\delta}^{\prime} \simeq D^{\prime} \cup \Sigma \sigma G_{k}$ and $M \simeq\left(D-\Sigma G_{k}\right)_{\bar{\sigma}}\left(D^{\prime} \smile \Sigma \sigma G_{k}\right)$. Similarly we have $M_{1-\delta} \simeq D-\Sigma G_{k}, M_{1-\delta}^{\prime} \simeq D^{\prime} \smile \Sigma \tau G_{k}$ and $M \simeq\left(D-\Sigma G_{k}\right) \smile\left(D^{\prime} \smile_{\Sigma_{\tau} G_{k}}\right)$. Since the boundary $\partial M_{t}$ is a submanifold of $M$ and moves continuously with respect to $t$ there exists a transformation of $M$ which map $M_{\delta}$ on $M_{1-\delta}$. We can assume without loss of generality that $\sigma G_{k}=\tau G_{k}$. And thus we have

Theorem. If $D_{\sigma}^{\smile} D^{\prime} \simeq D_{\tau}^{\smile} D^{\prime}$ then for a sufficiently large integer $m$ there exist transformation $y$ of $D-\sum_{i}^{m} G_{k}$ and transformation $z$ of $D^{\prime} \smile \sum_{1}^{m} \sigma G_{k}$ such that $\bar{\sigma} y=z \bar{\tau}$ where $\bar{\sigma}$ or $\bar{\tau}$ is the identifying map of $D-\Sigma G_{k}$ to $D^{\prime} \smile \Sigma_{\sigma} G_{k}$ obtained from $\sigma$ or $\tau$. 